\title{
Acoustic Guitar Playing System with Plucking Information Measured by 2 Dimensional Pickup
}

\author{
Ichiro TOKUHIRO ${ }^{1,2}$, Atsushi MIZUNO ${ }^{1}$, Yoshimitsu TAKASAWA ${ }^{3}$, Kiyohiko YAMAYA \\ ${ }^{1}$ Department of Information Media, Kanagawa Institute of Technology, Kanagawa, Japan \\ ${ }^{2}$ Showa University of Music, Kanagawa, Japan \\ ${ }^{3}$ Mirai Techno, Tokyo, Japan \\ ${ }^{4}$ Sona Research, Kanagawa, Japan
}

tokuhiro@ic.kanagawa-it.ac.jp

\begin{abstract}
The sound output from the audiovisual apparatus is inferior compared with the live performance. Information of music that the guitarist gave to the string can be measured by the 2 Dimensional Pickup devices we manufactured. Using the plucking information of guitar strings, we designed and constructed the Acoustic Guitar Playing System which can vibrate directly the bridge on the sound board of the guitar and can make guitar sound using the amplified power with the measured information of the 2 Dimensional Pickups.

To make a comparison with the sound between our Acoustic Guitar Playing System and the same acoustic guitar itself, we experimented with the aid of the sweep sound, the theoretical guitar synthesized sound and the flamenco music sound. The result of comparisons is that the sound of the Acoustic Guitar Playing System was superior in the low frequency range, but inferior in the high frequency range.
\end{abstract}

Keywords: Acoustic Guitar, 2 Dimensional Pickup, Plucking Information, Playing System,

\section{Introduction}

Music is said to be good for the mental health ${ }^{1)}$ and music also has the faculty to communicate together with the cultural exchange, such as Japan between South Korea, so music is indispensable in our life.

The sound output of the audiovisual apparatus is inferior compared with the live music performance. In researches on music from the point of academic views, large numbers of studies using sound analysis method about the sound played with musical instruments were presented $^{2)}$, but only small numbers of studies using the ergonomic approach such as the finger operation to play musical instruments were presented ${ }^{33}$.

This is the reason for a little ergonomic research on musical instruments, especially an acoustic guitar that there was no way to measure the guitarist's information such as the strength, the angle, the speed, and the direction of his fingers at his performance. Then we manufactured the 2 Dimensional Pickup ${ }^{4,5)}$ to measure the guitarist's information.

This paper presents The Acoustic Guitar Playing System with the plucking information measured by 2 Dimensional Pickup. Using the plucking information of guitar strings, we designed and constructed the Acoustic Guitar Playing System which can vibrate directly the bridge and can make the guitar sound using the amplified power of the measured information of 2 Dimensional Pickups.

To make a comparison with the sound between our Acoustic Guitar Playing System and the same acoustic guitar itself, we experimented with the aid of the sweep sound, the theoretical guitar synthesized sound and the flamenco music sound.

\section{2 Dimensional Pickup}

We designed and manufactured the 2 Dimensional Pickup which can measure the two dimensional force of the guitar string vibrating the bridge directly. Figure 1 shows the front view and the picture of the 2 Dimensional Pickup.
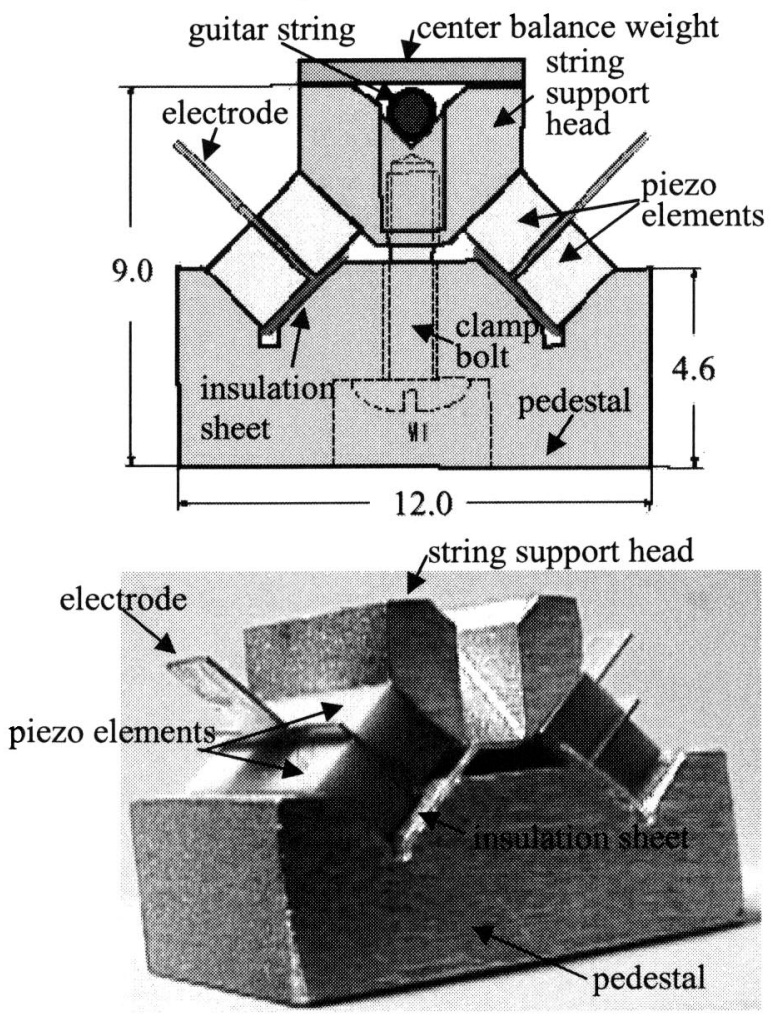

Figure1 2 Dimensional Pickup 
The 2 Dimensional Pickup was designed to measure the vibrating force of the bridge from $0.01[\mathrm{gw}]$ to 200 [gw] in two dimensions. The piezo electric element was C- 6 made by the Fuji Ceramic Company and had a size of $2 \times 6 \times 1.5$ [mm]. The 2 Dimensional Pickup is roughly consists of three parts which are the piezo elements, the string support head and the pedestal made from duralumin.

The string support head and the pedestal are firmly bound tight on both sides of the piezo electric element with the screw made from the stainless steel of M1.

\section{MIDI Guitar with the 2 Dimensional Pickups}

At the bridge part of the Kawai Classical Guitar, six arrayed 2 Dimensional Pickups were equipped under each six string. This guitar and its peripherals which can measure the guitarist's playing information of all strings were named "MIDI Guitar system". Figure 2 shows the connection between the MIDI guitar and peripherals

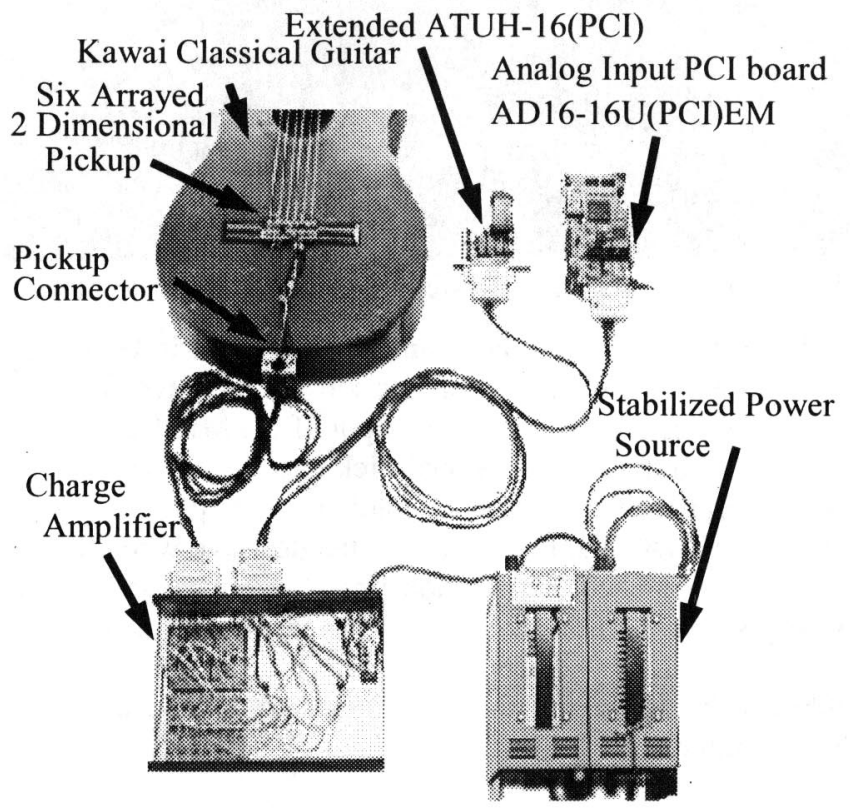

Figure 2 MIDI Guitar System
Six arrayed the 2 Dimensional Pickup are connected to the pickup connector at the bottom of the guitar which is connected to charge amplifier, and the output of the charge amplifier is inputted to the analog input PCI board AD16-16U EM (PCI) EM and Extended ATUH-16(PCI) which are inserted in the PCI slot of the personal computer.

Figure 3 shows a time waveform of the force vibrating vertically the bridge by the plucked guitar string when the guitarist played with the 3 succeed Rasgueado, which is one of the flamenco guitar performance method. Using a time waveform of both vertical and horizontal force, many information such as the strength, the angle, the speed and the direction of the guitarist's fingers on Rasgueado can be extracted.

Table 1 shows that the distance of the plucking position from the bridge at the $1^{\text {st }}$ to the $4^{\text {th }}$ string, the absolute angle to the standard which made the sound board of the guitar 0 degree, the plucking strength which define as the change of the vibrating force to the bridge per unit time and the timing which begins a start to measurement from the rising stroke of the thumb[p:pulgar] on the 1 st string.

Table 1. Plucking Information measured by MIDI Guitar

\begin{tabular}{c|c|c|c|c|c}
\hline \multirow{3}{*}{ String } & \multirow{2}{*}{ Finger } & position & $\mid$ angle $\mid$ & strength & timing \\
\cline { 2 - 6 } & & {$[\mathrm{cm}]$} & $\left.{ }^{\circ}\right]$ & {$[E U]$} & {$[\mathrm{msec}]$} \\
\hline \multirow{4}{*}{ 1st } & $p$ & 12.81 & 44.4 & 259 & 16.2 \\
\cline { 2 - 6 } & $c h$ & 8.22 & 43.1 & 184 & 130.7 \\
\cline { 2 - 6 } & $i$ & 12.81 & 29.6 & 1376 & 210.7 \\
\hline \multirow{4}{*}{ 2nd } & $p$ & 14.27 & 33.9 & 147 & 23.0 \\
\cline { 2 - 6 } & $c h$ & 8.16 & 39.5 & 208 & 126.1 \\
\cline { 2 - 6 } & $i$ & 13.06 & 17.1 & 1596 & 200.8 \\
\hline \multirow{4}{*}{ 3rd } & $p$ & 15.57 & 32.5 & 245 & 32.6 \\
\cline { 2 - 6 } & $c h$ & 8.01 & 39.7 & 299 & 114.6 \\
\cline { 2 - 6 } & $i$ & 14.27 & 25.5 & 1678 & 188.6 \\
\hline \multirow{3}{*}{ 4th } & $p$ & \multicolumn{4}{|c}{ no plucking } \\
\cline { 2 - 6 } & $c h$ & 7.88 & 60.7 & 580 & 101.1 \\
\cline { 2 - 6 } & $i$ & 16.37 & 64.1 & 323 & 183.3 \\
\hline
\end{tabular}

i:índice(index finger),ch:chica(little finger)

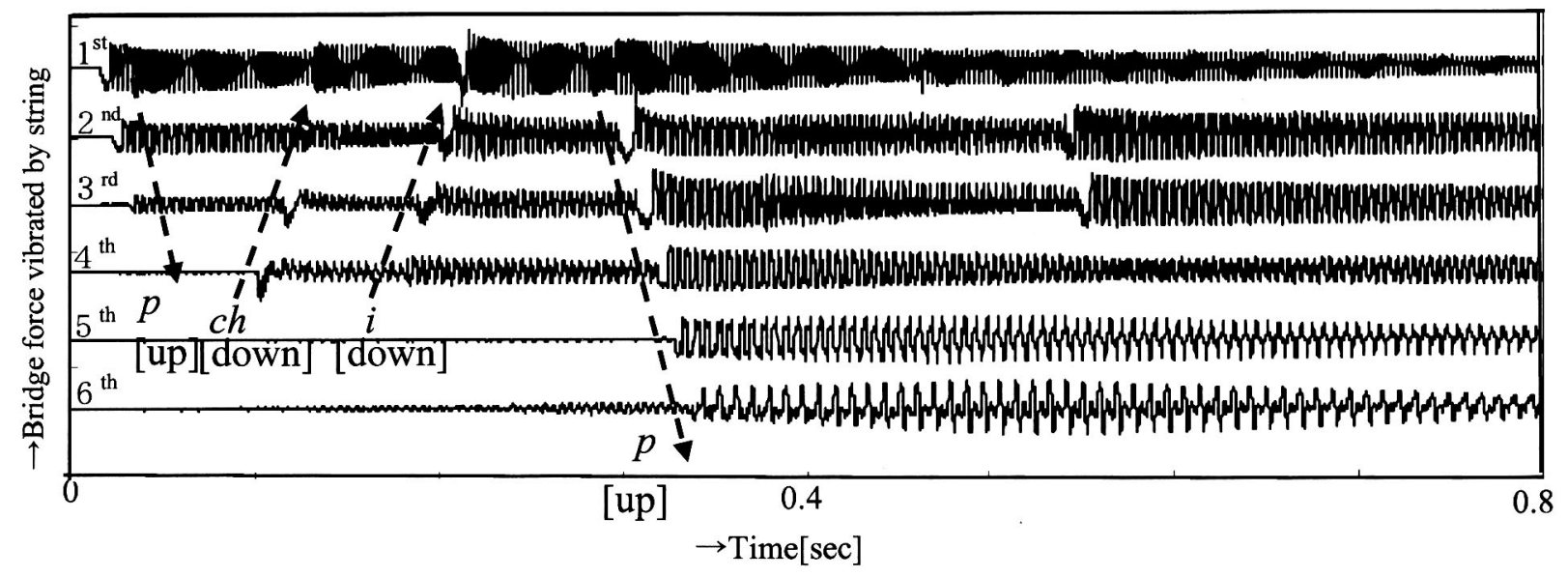

Figure 3 Bridge Force vibrated by String plucked with 3 succeed Rasgueado 


\section{Acoustic Guitar Playing System}

The sound output from the audiovisual apparatus is inferior compared with the live performance, so in order to enjoy live music without a human performance, many automatic performance systems, just like the YAMAHA silent ensemble piano model XP, were developed. By the aid of computer control using MIDI information, the guitar playing robot ${ }^{6)}$ was also manufactured. But the robot couldn't play enough well the guitar just like a guitarist. The reason why the unskillful performance by the robot is that the robot can not nowadays be build up to move perfect completely just like guitarist's hands and fingers.

So using the plucking information measured by MIDI Guitar system shown in Figure 2, we made up the Acoustic Guitar Playing System which can vibrate directly the bridge and can make sound from the guitar sound board with the aid of the amplified power of the measured information by the 2 Dimensional Pickup.

Figure 4 shows the Acoustic Guitar Playing System in which the vibration of the speaker drive unit connecting to the amplifier of the information measured by the 2 Dimensional Pickup is transmitted to the bridge on the surface sound board of the guitar directly through the vibration transmit aluminum rod.

The back sound board of the Kawai Classic Guitar was improved so that removal might be possible. The form of braces pasted on the surface sound board, which has great influences on the tone or the loudness, can be changed.

This Acoustic Guitar Playing System can play with both the output of the MIDI Guitar System and the music of CD or DVD.

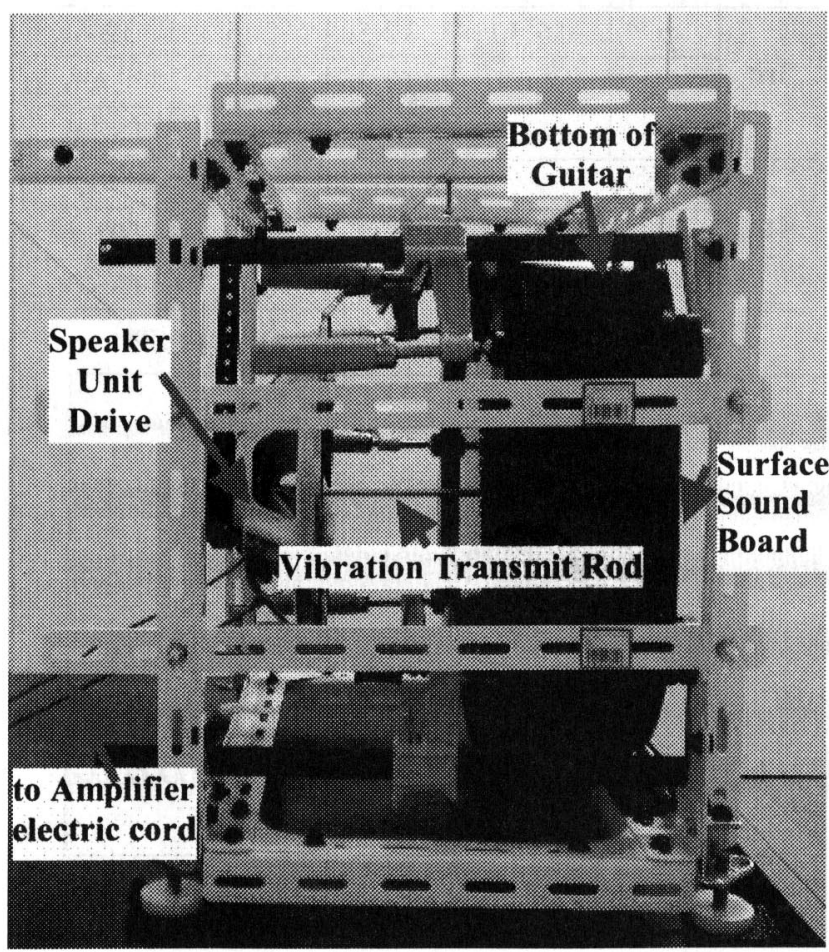

Figure 4 Guitar Playing System used Plucking Information

\section{Evaluation of Acoustic Guitar Playing System}

Under the conditions of the reverberating time 0.32 second at the listening laboratory shown in Figure 5, the sound from the Acoustic Guitar Playing System was recorded at the two positions by microphones. One is the optimal place ${ }^{6)}$ where the SONY microphone $\mathrm{C}-800 \mathrm{G}$ is put on $100 \mathrm{~cm}$ of 45 degrees to right direction from the guitar sound hole and the other is the normal listening position where the SONY microphone C-800 is put on $308 \mathrm{~cm}$ from front of the guitar.

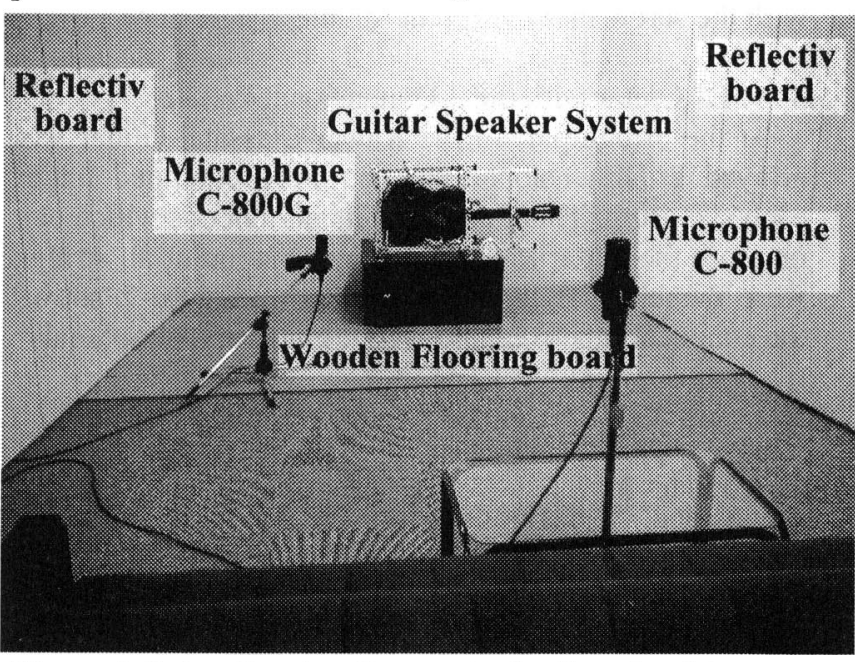

Figure 5 Guitar Speaker System in the listening laboratory

In order to evaluate the sound of the Acoustic Guitar Playing System with the comparison of the wave form and the spectrum, we took the output of the MIDI Guitar System with the 2 Dimensional Pickup when the G code for Taranto of a flamenco guitar was played by RASUGYADO, the theoretical rectangle wave when the string was plucked $1 / 7$ position from the bridge and the sweep sound from $20 \mathrm{~Hz}$ to $20 \mathrm{kHz}$, as the system driving sound source. Figure 6 showed the bridge force vibrated by string and the sound pressure level emitted from Guitar.

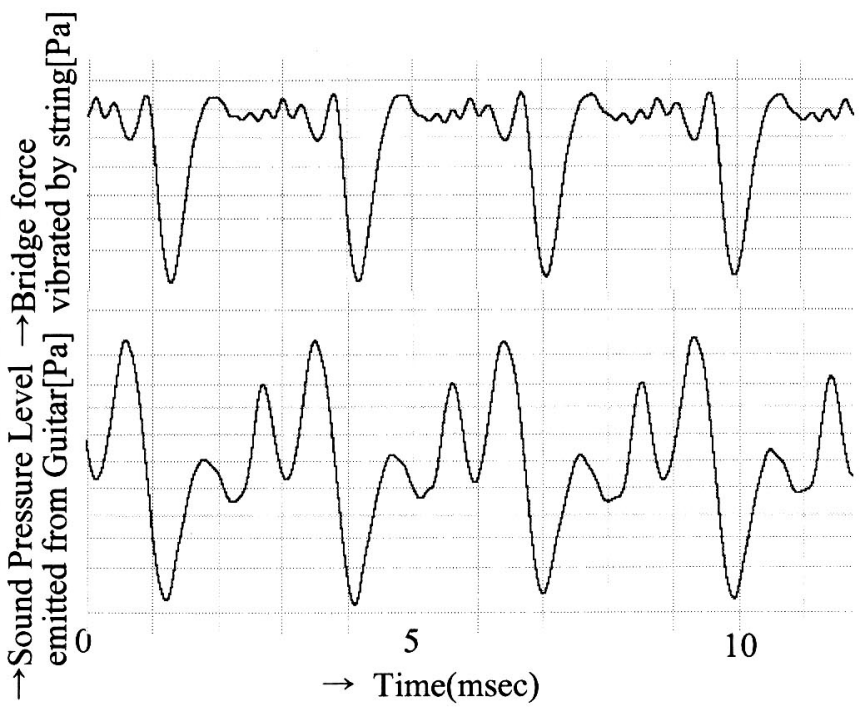

Figure 6 Bridge force vibrated by string and Sound Pressure Level emitted from Guitar 
It turns out that the rectangle wave form which vibrates a bridge on the surface sound board of the guitar is filtered and changed to the waveform peculiar to the guitar sound from Figure 6. The wavelet which exists on the rectangle wave is a part of a guitarist's information of the performance. The sound emitted from the guitar was able to be heard more softly than the sound which vibrated the bridge.

A $3^{\text {rd }}$ string is actually stretched with the guitar of the Guitar Playing System and the output sound of the 3rd plucking was recorded into the P.C.. The comparison with the sound spectrum between the guitar output and the guitar system is shown in Figure 7. It turns out that guitar system output sound shows a high spectrum value in a low frequency range compared with performance sound, and the low spectrum value is shown in a high frequency range from Figure 7.

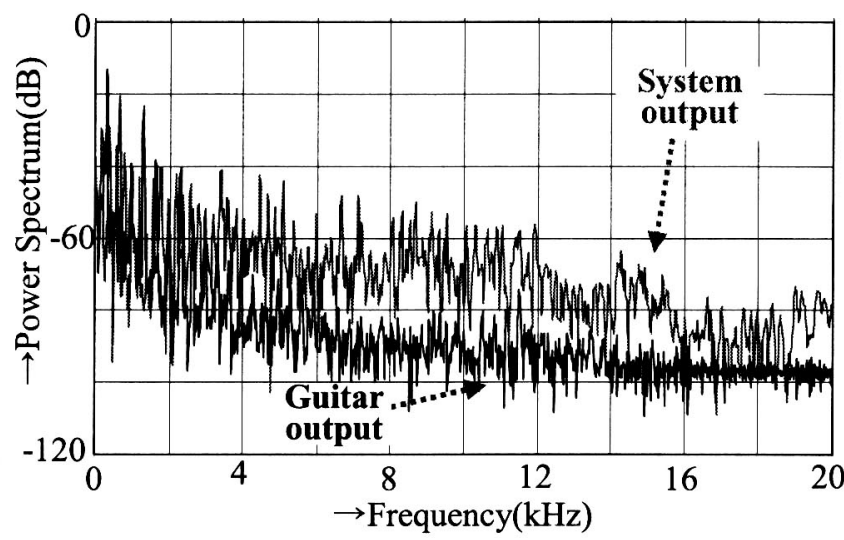

Figure 7 Spectrum of the guitar output and System output

Figure 8 shows the time waveform of system output sound which is the response to the sine wave sweep input of $20[\mathrm{~Hz}]$ to $20[\mathrm{kHz}]$ for 20 seconds. In Figure 8 it is clear that the Guitar Playing System has the high sensibility to the input of the guitar bridge. Since the number of time[sec] can be transposed to the number of frequency $[\mathrm{kHz}]$ in Figure 8 , the resonance frequency and its mode response were found clearly.

\section{Conclusion}

Using the plucking information of guitar strings, we designed and constructed the Acoustic Guitar Playing System which can vibrate directly the bridge on the sound board of the guitar and can make guitar sound using the amplified power with the measured information of the 2 Dimensional Pickups.

To make a comparison with the sound between our Acoustic Guitar Playing System between the same acoustic guitar itself, the sweep sound, the theoretical guitar synthesized and the flamenco music sound were used. It turns out that guitar system output sound shows a high spectrum value in a low frequency range compared with performance sound, and the low spectrum value is shown in a high frequency range.

\section{Acknowledgment}

Authors wish to thank Junpei KANNO who made the artificial finger and the plucking equipment, Hasumi TAKASHIMA who made the listening room with the variable reverberating time and Masaya HIKITA who made the TAKASAWA mode Speaker. They are the members of Tokuhiro Laboratory at Kanagawa Institute of Technology.

\section{References}

[1]American Music Therapy Association, Inc., http://www.musictherapy.org/

[2]Fletcher N.H., Rossing T.D.:The Physics of Musical Instruments, Springe1993

[3]Tokuhiro I., Deguchi T., Takasawa Y. and Yamaya K.: Element of Musical Instrument as Excellent One, Japan Ergonomics Society, The $46^{\text {th }}$ meeting, 2-3, 2005

[4]Tokuhiro I., Sato H., Nakashima K., Takasawa Y. and Yamaya K.: Pickup Development for 2 Dimensional Force Measurments of String Vibration at Supported terminal, MA00-3, Acoustical Society of Japan, 15-22, 2000

[5]Tokuhiro I., Miura T., Ninomiya K., Takasawa Y. and Yamaya K.: Differences of Force at Bridge give by some Plucking Methods and String with 2 Dimensional Pick-up, MA00-36, Acoustical Society of Japan, 1-8, 2000

[6]Tokuhiro I., Oshima N., Asano M. and Watanabe H.: Optimal Place to hear Sounds Played Flamenco guitar, MA97-7, Acoustical Society of Japan, 15-22, 1997

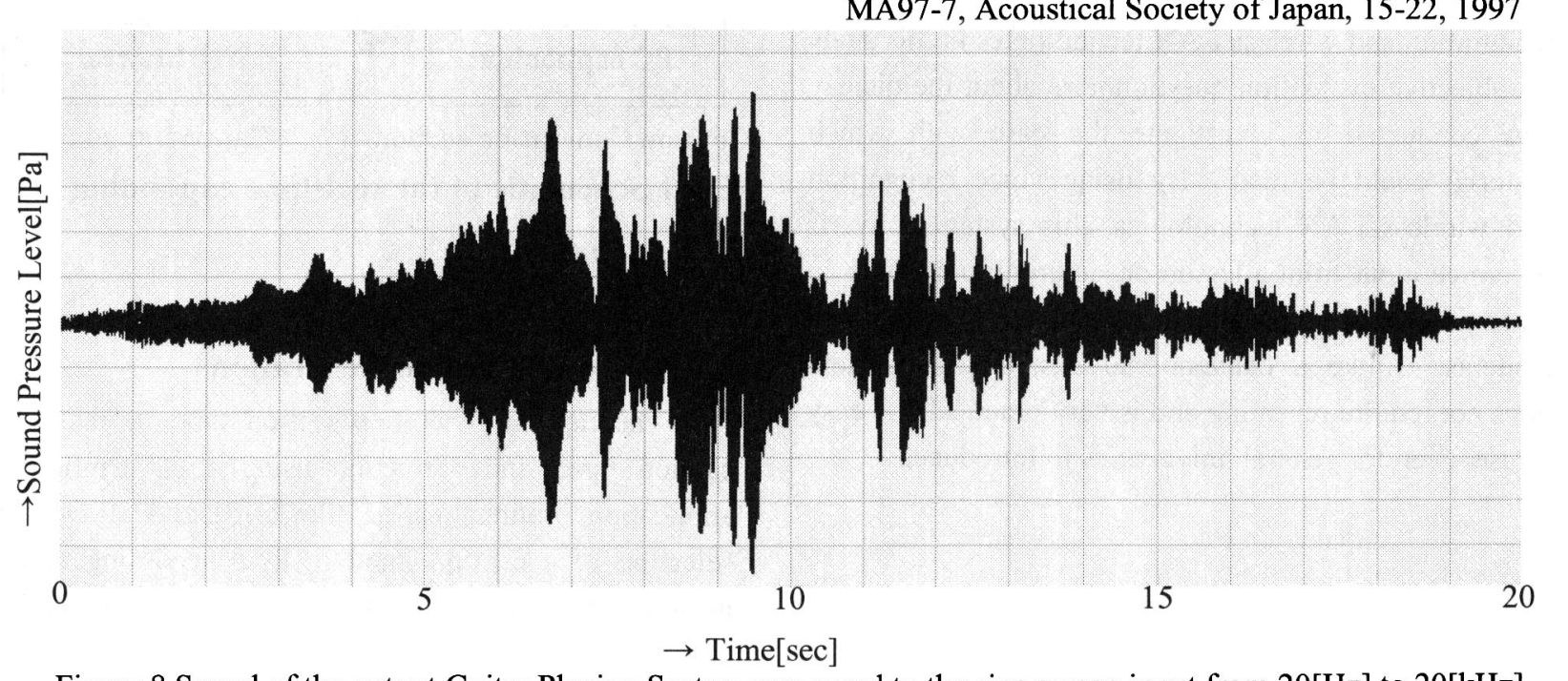

Figure 8 Sound of the output Guitar Playing System responsed to the sine sweep input from $20[\mathrm{~Hz}]$ to $20[\mathrm{kHz}]$. 